\title{
SUR LES REFLEXIONS DES COURBES DE LAVRENTIEV
}

\author{
MICHEL ZINSMEISTER
}

(Communicated by Albert Baernstein II)

\begin{abstract}
We answer a question of Semmes: A Jordan curve $\Gamma$ admitting a quasiconformal reflexion, which is "conformal" near $\Gamma$ in a sense given precisely by a Carleson measure condition, is necessarily rectifiable.
\end{abstract}

\section{INTRODUCTION ET ÉNONCÉ DU RÉSULTAT}

L'objet de cette note est de montrer comment les résultats de [AZ, Z] permettent de répondre à une question de Semmes.

Soit $\Gamma$ un quasicercle, c'est-à-dire l'image du cercle unité par une application quasiconforme de la sphère de Riemann $S^{2}$. Ahlfors [A] a montré que les quasicercles sont précisément les ensembles de points fixes des involutions quasiconformes de la sphère qui changent l'orientation (plus précisément une telle involution est nécessairement conjuguée à la symétrie par rapport à la droite réelle). Il a également montré que si $\Gamma$ contient le point à l'infini, cette reflexion peut être choisie bilipschitzienne pour la métrique euclidienne du plan.

Dire que la reflexion $f$ est quasiconforme revient à dire, dans le cas présent ( $f$ change l'orientation), que $f$ appartient à l'espace de Sobolev $H_{\mathrm{loc}}^{1}\left(S^{2}\right)$ et que

$$
\frac{\partial f}{\partial z}=\mu \frac{\partial f}{\partial \bar{z}}
$$

pour une fonction $\mu \in L^{\infty}\left(S^{2}\right),\|\mu\|_{\infty}<1$. Cette fonction, appelée la dilatation de $f$, mesure la déviation de la conformalité, $\mu=0$ sur un ouvert signifiant que $f$ y est antianalytique.

Dans [S] Semmes pose la question suivante:

Soit $\Gamma$ un quasicercle du plan admettant une reflexion quasiconforme de dilatation $\mu$ telle que

$$
\begin{gathered}
f \text { est }(1+\varepsilon) \text {-bilipschitzienne, } \\
\exists C>0 ; \forall z \in \Gamma, \forall r>0, \quad \iint_{D(z, r)} \frac{|\mu(\zeta)|^{2}}{\operatorname{dist}(\zeta, \Gamma)} d \zeta d \bar{\zeta} \leq \varepsilon r .
\end{gathered}
$$

Received by the editors May 11, 1992 and, in revised form, July 22, 1992.

1991 Mathematics Subject Classification. Primary 30C62; Secondary 42B99.

Ce travail a été subventionné en partie par le contrat NSF DMS-900 3438. 
Est-il vrai que $\Gamma$ est une courbe de Lavrentiev, c'est-à-dire l'image bilipschitzienne d'une droite ou d'un cercle, si $\varepsilon$ est assez petit?

Le résultat principal de ce travail est une réponse positive à cette question. Plus précisément, nous nous proposons de démontrer le

Théorème 1. Il existe $\varepsilon_{0}>0$ tel que tout quasicercle admettant une reflexion quasiconforme dont la dilatation vérifie (2) avec $\varepsilon \leq \varepsilon_{0}$ est une courbe de Lavrentiev (en particulier rectifiable).

L'auteur tient à remercier K. Astala, J. Heinonen, et S. Semmes pour leur aide, ainsi que $\mathrm{F}$. Gehring pour son invitation à l'université du Michigan où ce travail a été accompli.

\section{DÉMONSTRATION DU THÉORÈME 1}

Afin d'éviter les complications techniques, nous supposerons la courbe $\Gamma$ bornée et désignons par $\Omega$ la composante bornée de son complémentaire. Le cas général s'en déduit par des méthodes standard.

Soit $\varphi$ une représentation conforme du disque unité $D$ sur $\Omega$; on définit un prolongement quasiconforme de $\varphi$ à la sphère de Riemann par

$$
\forall z \in S^{2} \backslash \bar{D}, \quad \varphi(z)=f(\psi(z)), \quad \psi(z)=\varphi(1 / \bar{z})
$$

(on suppose sans restriction de généralité que $f(0)=\infty, \varphi(0)=0$ de sorte que $\varphi(\infty)=\infty)$. L'application $\varphi$ ainsi étendue conserve l'orientation et sa dilatation

$$
\nu=\frac{\partial \varphi}{\partial \bar{z}} / \frac{\partial \varphi}{\partial z}
$$

vérifie $|\nu(z)|=|\mu(\psi(z))|,|z|>1$.

Si $\mathscr{U}$ est un domaine du plan, on définit comme dans [Z2] l'espace $M C(\mathscr{U})$ des mesures de Carleson sur $\mathscr{U}$ par:

$$
\begin{aligned}
& m \in M C(\mathscr{U}) \\
& \quad \Leftrightarrow \sup \{|m|(D(z, r)) / r, \quad z \in \partial \mathscr{U}, 0<r<\operatorname{diam}(\mathscr{U})\}=\|m\|_{M C(U)}<+\infty .
\end{aligned}
$$

Notre première tâche va consister à estimer

$$
\left\|\frac{|\nu|^{2}}{|z|-r} d x d y\right\|_{M C(U(r))}
$$

où $U(r)$ désigne l'ouvert $\{|z|>r\}$ et $r$ est un réel $<1$.

Soit donc $z \in \mathbb{C},|z|=r$ et $\rho \leq 2 r$; si $\rho<1-r$, il n'y a rien à démontrer. Sinon, soit $\alpha$ une (petite) constante positive qui sera déterminée plus tard. Alors

$$
\begin{aligned}
\iint_{D(z, r)} \frac{|\nu|^{2}(\zeta)}{|\zeta|-r} d x d y & \leq 5 \alpha \rho+\iint_{D(z, \rho) \cap\{|\zeta|>1+\alpha(1-r)\}} \frac{|\nu|^{2}(\zeta)}{|\zeta|-r} d x d y \\
& =5 \alpha \rho+I(\rho) .
\end{aligned}
$$

On pose $\Omega_{r}=\varphi(\{|z|<r\}), r^{\prime}=1 /(1+\alpha(1-r))$, et $N(r)=\left\|\log \varphi^{\prime}\left(r e^{i t}\right)\right\|_{\mathrm{BMO}(T)}$.

Lemme 1. Il existe des constantes $C, K>0$ telles que

$$
I(\rho) \leq C \sqrt{\varepsilon} \alpha^{-K} e^{C N(r)} \rho .
$$


Preuve. Par le théorème de distorsion de Koebe,

$$
\frac{|\nu|^{2}(\zeta)}{|\zeta|-r} \leq \frac{|\nu|^{2}(\zeta)}{|\zeta|-1} \leq c \frac{|\mu(\psi(\zeta))|^{2}}{\operatorname{dist}(\psi(\zeta), \Gamma)}\left|\frac{\partial \psi}{\partial \bar{z}}(\zeta)\right| .
$$

On montre ensuite que

$$
\left\|\frac{|\mu|^{2}}{\operatorname{dist}(\cdot, \Gamma)}\right\|_{M C\left(\Omega\left(r^{\prime}\right)\right)} \leq C \sqrt{\varepsilon}
$$

pour le voir, on teste sur les disques $D(\omega, \eta)$ avec $\omega \in \partial \Omega\left(r^{\prime}\right)$. Si $\eta<$ $\sqrt{\varepsilon} \operatorname{dist}(\omega, \Gamma)$, une estimation triviale permet de conclure. Sinon $D(\omega, \eta)$ est inclus dans un disque centré sur $\Gamma$ et de rayon $2 \eta / \sqrt{\varepsilon}$ et l'on conclut en appliquant (2). De (3) et de cette dernière estimation, une application du théorème principal de [Z2] permet d'obtenir

$$
I(\rho) \leq C \sqrt{\varepsilon} \rho e^{C N\left(r^{\prime}\right)}
$$

et donc le lemme 1 car le théorème de distorsion de Koebe implique

$$
N\left(r^{\prime}\right) \leq N(r)+C \log (1 / \alpha)
$$

Supposons dans un premier temps que $N(r) \leq M$, une constante à déterminer. Si $S_{\varphi}$ désigne la dérivée schwarzienne de $\varphi$ et

$$
S(r)=\left\|(r-|z|)^{3}\left|S_{\varphi}(z)\right|^{2}\right\|_{M C(D(0, r))},
$$

alors un des résultats de $[A Z]$ implique que

$$
S(r) \leq C\left\|\frac{|\nu|^{2}}{|z|-r}\right\|_{M C(U(r))} \leq C\left(\alpha^{-K} \sqrt{\varepsilon} e^{C M}+\alpha\right),
$$

la deuxième inégalité découlant du lemme 1. Par un autre résultat de $[\mathrm{AZ}]$, il existe une constante $A>0$ telle que $N(r) \leq A(S(r)+1)$. Enfin, un résultat "classique" (voir, par exemple, [Z1]) affirme l'existence d'un $\beta>0$ tel que $\varphi(D)$ est un domaine de Lavrentiev si $S(1)<\beta$.

On raisonne alors comme suit: On commence par choisir $M>2 A(1+\beta)$ puis $\alpha<\beta / 4 C$ et enfin $\varepsilon$ de sorte que le troisième membre de (4) soit $<\beta / 2$.

Soit alors $E$ l'ensemble des $r \in[0,1)$ tels que $N(t) \leq M$ pour $t \leq r: E$ est fermé non vide car $N$ est une fonction continue qui tend vers 0 en 0 . Les résultats précédents impliquent également que c'est un ouvert car, par (4) et le choix des constantes,

$$
N(r) \leq M \Rightarrow S(r)<\frac{\beta}{2} \quad \text { et } \quad N(r) \leq \frac{M}{2} .
$$

Donc $E=[0,1), S(r) \leq \beta / 2$ si $r<1$ et par conséquent $S(1)<\beta$ par le lemme de Fatou, ce qui achève la preuve du théorème.

\section{REFERENCES}

[A] L. Ahlfors, Quasiconformal reflections, Acta Math. 109 (1963), 291-301.

[AZ] K. Astala and M. Zinsmeister, Teichmüller spaces and BMOA, Math. Ann. 289 (1991), 613-625. 
[S] S. Semmes, Chord-arc surfaces with small constant II: Good parameterizations, Adv. Math. 88 (1991), 170-199.

[Z1] M. Zinsmeister, Représentation conforme et courbes presque lipschitziennes, Ann. Inst. Fourier (Grenoble) 34 (1984), 29-44.

[Z2] L Les domaines de Carleson, Michigan Math. J. 36 (1989), 213-220.

Department of Mathematics, The University of Michigan, Ann Arbor, Michigan 48109 Current address: Département de Mathématique, Université d'Orléans, BP 6749 F-45067 Orléans Cedex 2, France

E-mail address: zins@Labomath.univ-orleans.fr 